\title{
Gravity and the Quantum: Are they Reconcilable?
}

\author{
R. Aldrovandi*, J. G. Pereira* and K. H. Vu* \\ * Instituto de Física Teórica, Universidade Estadual Paulista \\ Rua Pamplona 145, 01405-900 São Paulo, Brazil
}

\begin{abstract}
General relativity and quantum mechanics are not consistent with each other. This conflict stems from the very fundamental principles on which these theories are grounded. General relativity, on one hand, is based on the equivalence principle, whose strong version establishes the local equivalence between gravitation and inertia. Quantum mechanics, on the other hand, is fundamentally based on the uncertainty principle, which is essentially nonlocal. This difference precludes the existence of a quantum version of the strong equivalence principle, and consequently of a quantum version of general relativity. Furthermore, there are compelling experimental evidences that a quantum object in the presence of a gravitational field violates the weak equivalence principle. Now it so happens that, in addition to general relativity, gravitation has an alternative, though equivalent, description, given by teleparallel gravity, a gauge theory for the translation group. In this theory torsion, instead of curvature, is assumed to represent the gravitational field. These two descriptions lead to the same classical results, but are conceptually different. In general relativity, curvature geometrizes the interaction while torsion, in teleparallel gravity, acts as a force, similar to the Lorentz force of electrodynamics. Because of this peculiar property, teleparallel gravity describes the gravitational interaction without requiring any of the equivalence principle versions. The replacement of general relativity by teleparallel gravity may, in consequence, lead to a conceptual reconciliation of gravitation with quantum mechanics.
\end{abstract}

Keywords: Gravitation, Quantum Mechanics, Teleparallel Gravity

PACS: 04.20.Cv; 04.50.+h

\section{INTRODUCTION}

\subsection{General Relativity and Universality}

At least at the classical level, gravitation shows a quite peculiar property: particles with different masses and different compositions feel it in such a way that all of them acquire the same acceleration and, given the same initial conditions, follow the same path. Such universality of response-usually referred to as universality of free fall-is the most fundamental characteristic of the gravitational interaction [1]. It is unique, peculiar to gravitation: no other basic interaction of Nature has it. Effects equally felt by all bodies were known since long. They are the so called inertial effects, which show up in non-inertial frames. Examples on Earth are the centrifugal and the Coriolis forces.

Universality of inertial effects was one of the clues used by Einstein in building up general relativity, his theory for gravitation. Another ingredient was the notion of field. That concept provides the best approach to interactions coherent with special relativity. All known forces are mediated by fields on spacetime. If gravitation is to be represented by a field, it should, by the considerations above, be a universal field, equally felt by every particle. A natural solution is to assume that gravitation changes spacetime itself. And, of all the fields present in a spacetime, the metric appears as the most fundamental. The simplest way to change spacetime, then, would be to change its metric. Furthermore, the metric does change when looked at from a non-inertial frame, in which case the (also universal) inertial effects are present. The presence of a gravitational field should be, therefore, represented by a change in the spacetime metric. In absence of gravitation that metric should reduce to the flat Minkowski metric.

A crucial point in this description, which is fundamentally based on the universality of free fall, is that it makes no use of the concept of force for the gravitational interaction. In fact, instead of acting through a force, gravitation is represented by a deformation of the spacetime structure. More precisely, the presence of a gravitational field is supposed to produce a curvature in spacetime, a (spinless) particle in a gravitational field simply follows a geodesics of the modified spacetime. Notice that no other kind of spacetime deformation is supposed to exist. Torsion, for example, which would be another natural spacetime deformation, is assumed to vanish from the very beginning. This is the approach of general relativity, in which geometry replaces the concept of gravitational force, and the trajectories are determined, not by force equations, but by geodesics. The underlying spacetimes are pseudo-Riemannian spaces. It is important to remark that only an interaction presenting the property of universality can be described by such a 
geometrization of spacetime. In the eventual absence of universality, the general relativity description of gravitation would break down.

It is important to observe that universality of free fall is usually identified as the statement of the weak equivalence principle. In fact, if all particles move along geodesics, the motion must be independent of their masses, and consequently universal. Furthermore, in order to be independent of the mass, it must be somehow canceled out from the equation of motion. Since this cancellation can only be made when the inertial and gravitational masses coincide, this last statement is also usually identified with the weak equivalence principle. It should be remarked, however, that this is true only at the classical level. At the quantum level, as we are going to see, even if the inertial and gravitational masses coincide, the gravitational effects on quantum objects can still be mass-dependent.

\subsection{The Equivalence Versus the Uncertainty Principle}

General relativity and quantum mechanics are not consistent with each other. The seeds of discord are the very principles on which these theories take their roots. General relativity, on one hand, is based on the equivalence principle, whose strong version establishes the local equivalence between gravitation and inertia. The fundamental asset of quantum mechanics, on the other hand, is the uncertainty principle, which is essentially nonlocal: a precise localization of a test particle would lead to a infinite uncertainty in its momentum [2].

We may ask ourselves whether there is a real inconsistency, or a simple lack of common ground. The strong version of the equivalence principle, which implies the weak one, presupposes an ideal observer [3], represented by a timelike curve which intersects the space-section at a point. In each space-section, it applies at that intersecting point. The conflict comes, for the strong principle, from that idealization and extends, clearly, also to special relativity. In the equation for a curve, gravitation only appears through the Levi-Civita connection, which can be made to vanish all along. An ideal observer can choose frames whose acceleration exactly compensate the effect of gravitation. A real observer, on the other hand, will be necessarily an object extended in space, consequently intersecting a congruence of curves. Such congruences are described by the deviation equation and, consequently, detect the true covariant object characterizing the gravitational field, the curvature tensor which cannot be made to vanish. Quantum Mechanics requires real observers, pencils of ideal observers. The inconsistency with the strong principle, therefore, is a mathematical necessity.

A crucial question then arises: is there a peaceful way of reconciling the equivalence and the uncertainty principles? The answer seems to be no as these two principles are fundamentally different, and like darkness and lightness, they cannot hold simultaneously. It then comes the inevitable question: which one is to be discarded? Well, at first sight the answer seems to be very difficult because general relativity and quantum mechanics are two of the main pillars of modern physics, and discarding one of their underlying principles would mean to discard one of these pillars. However, a more careful analysis of this question strongly suggests that the equivalence principle is the weaker part of the building. In fact, there is a general agreement that, due to their intrinsic incompatibilities, it is not possible to construct a quantum version of the strong equivalence principle. Actually, even at the classical level, there are many controversies related with the correct meaning of its statements [4]. For example, in the Preface of his classic textbook [5], Synge confess that ... I have never been able to understand this Principle. Does it mean that the signature of the space-time metric is +2 (or -2 if you prefer the other convention)? If so, it is important, but hardly a Principle. Does it mean that the effects of a gravitational field are indistinguishable from the effects of an observer's acceleration? If so, it is false. In Einstein's theory, either there is a gravitational field or there is none, according to as the Riemann tensor does not or does vanish. This is an absolute property; it has nothing to do with any observer's world line. Space-time is either flat or curved, and in several places in the book I have been at considerable pains to separate truly gravitational effects due to curvature of space-time from those due to curvature of the observer's world-line (in most ordinary cases the latter predominate). The Principle of Equivalence performed the essential office of midwife at the birth of general relativity, but, as Einstein remarked, the infant would never have got beyond its long-clothes had it not been for Minkowski's concept. I suggest that the midwife be now buried with appropriate honours and the facts of absolute space-time faced. On the other hand, the inconsistency of quantum mechanics with the weak equivalence principle is a matter of experiment. Although it has passed all experimental tests at the classical level [6], there are compelling evidences that the weak equivalence principle might not be true at the quantum level.

Following the above arguments, the basic purpose of this paper will be to explore further the conceptual inconsistencies of general relativity with quantum mechanics. Then, by relying on this study, a possible way of reconciling gravitation with quantum mechanics will be proposed and analyzed. We begin by presenting, in the next section, some 
evidences that the weak equivalence principle fails at the quantum level.

\section{QUANTUM EFFECTS IN GRAVITATION}

There are in the literature some very simple idealized examples of possible quantum violations of the weak equivalence principle [7]. Here, however, we will present two very specific evidences, the first of them extensively verified experimentally. It is the so called Colella-Overhauser-Werner (COW) phenomenon [8]. It consists in using a neutron interferometer to observe the quantum mechanical phase shift of neutrons caused by their interaction with Earth's gravitational field, assumed to be Newtonian. Figure 1 shows a scheme of the experiment, which is performed in the presence of a Newtonian potential

$$
\phi \equiv g z
$$

where $g$ is the gravitational acceleration, supposed not to change significantly in the region of the experience, and $z$ is the distance from some reference point on Earth. In the presence of a gravitational field, because the segments BD and CE are at different distance from Earth, and consequently at different value of the potential $\phi$, there will be a gravitationally induced quantum phase shift between the two trajectories when they arrive at the screen. This phase shift is given by [8]

$$
\delta \varphi \equiv \varphi_{\mathrm{BCE}}-\varphi_{\mathrm{BDE}}=\frac{g r s}{\hbar v} m,
$$

where $r$ and $s$ are dimensions of the interferometer (see Fig. 1), $v$ is the velocity, and $m$ is the mass of the neutron. From this expression we can see that the quantum phase difference induced by the gravitational field depends explicitly on the mass of the particle. More specifically, if we distinct the gravitational $\left(m_{g}\right)$ and inertial $\left(m_{i}\right)$ masses, the phase difference in this case would be $[9,10]$

$$
\delta \varphi=\frac{g r s}{\hbar v} m_{g}
$$

from where we see that, actually, the phase shift depends on the gravitational mass of the particle. At the quantum level, therefore, due to this dependence, gravitation seems to be no more universal [11]. ${ }^{1}$

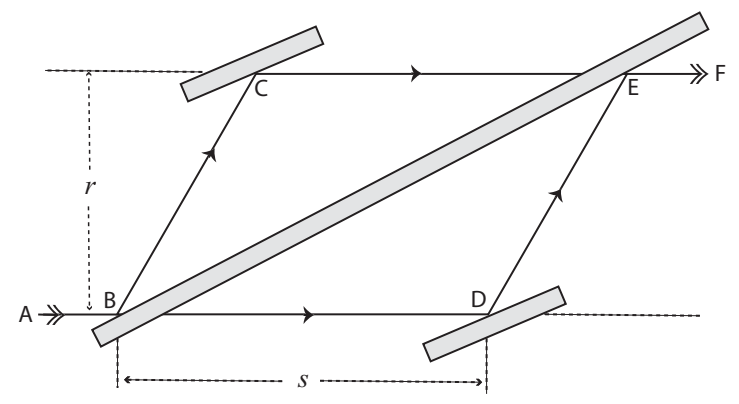

FIGURE 1. Schematic illustration of the COW neutron interferometer.

As another evidence of a possible quantum violation of universality, let us consider now the gravitational analog of the Aharonov-Bohm effect [13]. The usual (electromagnetic) Aharonov-Bohm effect consists in a shift, by a constant amount, of the electron interferometry wave pattern, in a region where there is no magnetic field, but there is a nontrivial electromagnetic potential. Analogously, the gravitational Aharonov-Bohm effect will consist in a similar shift of the same wave pattern, but produced by the presence of a gravitational potential, in a region where there is no gravitational field. Phenomenologically, this kind of effect might be present near a massive rapidly rotating source, like a neutron star, for example. Of course, differently from an ideal apparatus, in a real situation the gravitational field cannot be completely eliminated, and consequently the gravitational Aharonov-Bohm effect should be added to the other effects also causing a phase change.

\footnotetext{
${ }^{1}$ It should be remarked that, through the introduction of a quantum version of the weak equivalence principle [12], the phase shift of non-relativistic interferometry experiments can be made independent of the mass if written in an appropriate way. This quantum principle, however, seems to be more operational than conceptual. Furthermore, it is not clear whether it remains valid in the relativistic domain.
} 


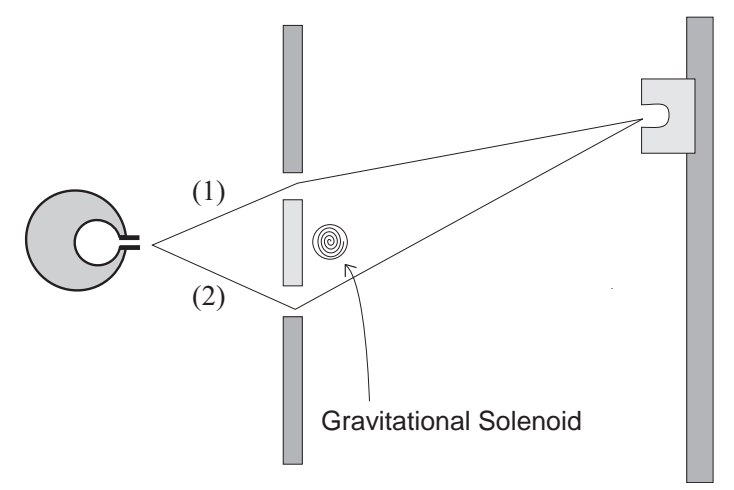

FIGURE 2. Schematic illustration of the Aharonov-Bohm electron interferometer.

We consider then the ideal case in which a kind of infinite "gravitational solenoid" produces a purely static gravitomagnetic field flux concentrated in its interior (see Fig. 2). In the ideal situation, the gravitational field outside the solenoid vanishes completely, but there is a nontrivial gravitational potential. When we let the electrons to move outside the solenoid, phase factors corresponding to paths lying on one side of the solenoid will interfere with phase factors corresponding to paths lying on the other side, which will produce an additional phase shift at the screen. Denoting by

$$
\Omega=\oint \vec{H} \cdot d \vec{\sigma}
$$

the flux of the gravitomagnetic field $\vec{H}$ inside the solenoid, the phase shift is found to be [9]

$$
\delta \varphi \equiv \varphi_{(2)}-\varphi_{(1)}=\frac{\mathscr{E} \Omega}{\hbar c},
$$

where $\mathscr{E}=\gamma m c^{2}$ is the electron kinetic energy, with $\gamma \equiv\left[1-\left(v^{2} / c^{2}\right)\right]^{-1 / 2}$ the relativistic factor. As it depends on the energy, this phase difference applies equally to massive and massless particles. For the case of massive particles, if we distinct gravitational and inertial masses, the phase shift would be [10]

$$
\delta \varphi=\frac{\mathscr{E} \Omega}{\hbar c}\left(\frac{m_{g}}{m_{i}}\right)=\frac{\gamma_{c} \Omega}{\hbar} m_{g}
$$

where now $\mathscr{E}=\gamma m_{i} c^{2}$. We see from this expression that, also in the gravitational Aharonov-Bohm effect, the phase shift depends on the (gravitational) mass of the particle. This is one more indication that, at the quantum level, gravitation seems to be no more universal.

\section{TELEPARALLEL GRAVITY}

\subsection{Can We Dispense with the Weak Equivalence Principle?}

The basic conclusion of the previous section was that there are strong indications that gravitation is no more universal at the quantum level. This means essentially that the weak equivalence principle is no more applicable at this level. However, as already discussed, without this principle, the geometrical description of general relativity breaks down. A new question then arises: are we able to manage without the equivalence principle, and consequently without general relativity? The remaining of this paper will be devoted to answer this question.

To begin with, let us remark that, like the other fundamental interactions of nature, gravitation can also be described in terms of a gauge theory [14]. In fact, the teleparallel equivalent of general relativity, or teleparallel gravity for 
short, ${ }^{2}$ can be interpreted as a gauge theory for the translation group. In this theory, instead of torsion, curvature is assumed to vanish. The corresponding underlying spacetime is, in this case, a Weitzenböck spacetime [16]. In spite of this fundamental difference, the two theories are found to yield equivalent classical descriptions of the gravitational interaction [17]. Conceptual differences, however, show up. According to general relativity, curvature is used to geometrize spacetime. Teleparallelism, on the other hand, attributes gravitation to torsion, but in this case torsion accounts for gravitation not by geometrizing the interaction, but by acting as a force. As a consequence, there are no geodesics in teleparallel gravity, but only force equations quite analogous to the Lorentz force equation of electrodynamics [18]. We may then say that the gravitational interaction can be described in terms of curvature, as is usually done in general relativity, or alternatively in terms of torsion, in which case we have the so called teleparallel gravity. Whether gravitation requires a curved or a torsioned connection—or equivalently, a Riemann or a Weitzenböck spacetime structure - turns out to be, at least classically, a matter of convention.

Now, as is widely known, the electromagnetic interaction is not universal: there exists no electromagnetic equivalence principle. As both Maxwell's theory and teleparallel gravity are Abelian gauge theories, in which the equations of motion of test particles are not geodesic but force equations, the question arises whether the gauge approach of teleparallel gravity would also be able to describe the gravitational interaction in the eventual lack of universality. As we are going to see, the answer to this question is yes - teleparallel gravity does not require the validity of the equivalence principle to describe the gravitational interaction [19]. Whereas the geometrical description of general relativity breaks down in the absence of universality, teleparallel gravity remains a consistent theory. In spite of the equivalence with general relativity, therefore, teleparallel gravity seems to belong to a more general class of theory. In order to understand this point, it is necessary first to study the fundamentals of teleparallel gravity.

\subsection{Fundamentals of Teleparallel Gravity}

The mathematical structure of distant parallelism, also referred to as absolute or teleparallelism, was used by Einstein in the late nineteen twenties, in his attempt to unify gravitation with electromagnetism. The crucial idea was the introduction of a tetrad field, a field of orthonormal bases of the tangent spaces at each point of the fourdimensional spacetime. The specification of a tetrad involves sixteen components, whereas the gravitational field, represented by the spacetime metric, requires only ten components. The six additional degrees of freedom ensued by the tetrad was then supposed by Einstein to represent the electromagnetic field. This attempt of unification did not succeed, but some of the concepts introduced by him remain important up to the present day [20].

According to the gauge structure of teleparallel gravity, to each point of spacetime there is attached a Minkowski tangent space, on which the translation (gauge) group acts. We use the Greek alphabet $\mu, v, \rho, \ldots=0,1,2,3$ to denote spacetime indices and the Latin alphabet $a, b, c, \ldots=0,1,2,3$ to denote algebraic indices related to the tangent Minkowski spaces, whose metric is chosen to be $\eta_{a b}=\operatorname{diag}(+1,-1,-1,-1)$. As a gauge theory for translations, the fundamental field of teleparallel gravity is the translational gauge potential $B^{a}{ }_{\mu}$, a 1-form assuming values in the Lie algebra of the translation group [18]

$$
B_{\mu}=B^{a}{ }_{\mu} P_{a},
$$

with $P_{a}=\partial_{a}$ the generators of infinitesimal translations. Under a local translation of the tangent space coordinates $\delta x^{a}=\varepsilon^{a}(x) \equiv \varepsilon^{a}$, the gauge potential transforms according to

$$
B^{\prime a}{ }_{\mu}=B^{a}{ }_{\mu}-\partial_{\mu} \varepsilon^{a} .
$$

It appears naturally as the nontrivial part of the tetrad field $h^{a}{ }_{\mu}$ :

$$
h^{a}{ }_{\mu}=\partial_{\mu} x^{a}+B^{a}{ }_{\mu} .
$$

If the tangent space indices are raised and lowered with the Minkowski metric $\eta_{a b}$, therefore, the spacetime indices will raised and lowered with the spacetime metric

$$
g_{\mu \nu}=\eta_{a b} h^{a}{ }_{\mu} h^{b} .
$$

\footnotetext{
2 The name teleparallel gravity is normally used to designate a theory in which there are three free parameters (see, for example, Ref. [15], and references therein). Here, however, we use it as a synonymous of the teleparallel equivalent of general relativity, a theory obtained for a specific choice of these parameters.
} 
The above tetrad can be used to construct the so called Weitzenböck connection

$$
\dot{\Gamma}^{\rho}{ }_{\mu v}=h_{a}{ }^{\rho} \partial_{v} h_{\mu}^{a},
$$

which introduces the distant parallelism in the four-dimensional spacetime manifold. It is a connection presenting torsion, but no curvature. Its torsion,

$$
\dot{T}^{\rho}{ }_{\mu \nu}=\dot{\Gamma}^{\rho}{ }_{v \mu}-\dot{\Gamma}^{\rho}{ }_{\mu \nu},
$$

is nothing but the translational gauge field strength $\dot{T}^{a}{ }_{\mu \nu}$, as seen from the tetrad frame:

$$
\dot{T}^{a}{ }_{\mu \nu} \equiv \partial_{\mu} B^{a}{ }_{\nu}-\partial_{\nu} B^{a}{ }_{\mu}=h^{a}{ }_{\rho} \dot{T}^{\rho}{ }_{\mu \nu} .
$$

The Weitzenböck connection is related to the Levi-Civita connection

$$
\stackrel{\circ}{\Gamma}_{\mu \nu}^{\rho}=\frac{1}{2} g^{\rho \sigma}\left(\partial_{\mu} g_{\sigma v}+\partial_{\nu} g_{\sigma \mu}-\partial_{\sigma} g_{\mu v}\right)
$$

of the spacetime metric $g_{\mu v}$ through

$$
\stackrel{\circ}{\Gamma}_{\mu v}=\stackrel{\circ}{\Gamma}_{\mu v}+\dot{\circ}^{\rho} \mu v
$$

where

$$
\dot{K}^{\rho}{ }_{\mu \nu}=\frac{1}{2}\left(\dot{T}_{\mu} \rho_{v}+\dot{T}_{v} \rho_{\mu}-\dot{T}^{\rho}{ }_{\mu \nu}\right)
$$

is the contortion tensor.

The Lagrangian of the teleparallel equivalent of general relativity is [18]

$$
\dot{\mathscr{L}}=\frac{h}{8 k^{2}}\left[\dot{T}^{\rho}{ }_{\mu \nu} \dot{T}_{\rho}^{\mu \nu}+2 \dot{T}^{\rho}{ }_{\mu \nu} \dot{T}^{v \mu}{ }_{\rho}-4 \dot{T}_{\rho \mu}{ }^{\rho} \dot{T}^{v \mu}{ }_{v}\right] .
$$

where $k^{2}=8 \pi G / c^{4}$ and $h \equiv \sqrt{-g}=\operatorname{det}\left(h^{a}{ }_{\mu}\right)$, with $g=\operatorname{det}\left(g_{\mu \nu}\right)$. The first term corresponds to the usual Lagrangian of internal, or Yang-Mills gauge theories. In the gravitational case, however, owing to the presence of a tetrad field, which are components of the solder form [21], algebra and spacetime indices can be changed into each other, and in consequence new contractions turn out to be possible. It is exactly this possibility that gives rise to the other two terms of the above Lagrangian. Defining the tensor

$$
\dot{S}^{\rho \mu v}=-\dot{S}^{\rho v \mu}=\left[\dot{K}^{\mu v \rho}-g^{\rho v} \dot{T}^{\sigma \mu}{ }_{\sigma}+g^{\rho \mu} \dot{T}_{\sigma}^{\sigma v}\right],
$$

usually called superpotential [22], it can be rewritten in the form [23]

$$
\dot{\mathscr{L}}=\frac{h}{4 k^{2}} \dot{T}^{\rho}{ }_{\mu \nu} \dot{S}_{\rho}{ }^{\mu v} .
$$

Let us consider now the Lagrangian

$$
\mathscr{L}=\dot{\mathscr{L}}+\mathscr{L}_{m}
$$

where $\mathscr{L}_{m}$ represents the Lagrangian of a general matter field. By performing variations in relation to the gauge field $B^{a}{ }_{\rho}$, we obtain the teleparallel version of the gravitational field equation

$$
\partial_{\sigma}\left(h \dot{S}_{a}{ }^{\rho \sigma}\right)-k^{2}\left(h \dot{j}_{a}{ }^{\rho}\right)=k^{2}\left(h \mathscr{T}_{a}^{\rho}\right)
$$

where $\dot{S}_{a}{ }^{\rho \sigma}=h_{a}{ }^{\lambda} \dot{S}_{\lambda} \rho \sigma$, the current

$$
\dot{j}_{a}{ }^{\rho} \equiv-\frac{\partial \dot{\mathscr{L}}}{\partial h^{a}{ }_{\rho}}=\frac{h_{a}^{\lambda}}{k^{2}}\left(\dot{T}^{c}{ }_{\mu \lambda} \dot{S}_{c}{ }^{\mu \rho}-\frac{1}{4} \delta_{\lambda}{ }^{\rho}{\dot{T^{c}}}^{c}{ }_{\mu \nu}{\dot{S_{c}}}^{\mu \nu}\right)
$$

represents the tensorial form of the gravitational energy-momentum density [24], and

$$
h \mathscr{T}_{a}^{\rho} \equiv-\frac{\delta \mathscr{L}_{m}}{\delta B^{a}{ }_{\rho}} \equiv-\frac{\delta \mathscr{L}_{m}}{\delta h^{a} \rho}=-\left(\frac{\partial \mathscr{L}_{m}}{\partial h^{a}{ }_{\rho}}-\partial_{\lambda} \frac{\partial \mathscr{L}_{m}}{\partial_{\lambda} \partial h^{a}{ }_{\rho}}\right)
$$


is the matter energy-momentum tensor. Due to the anti-symmetry of $\dot{S}_{a} \rho \sigma$ in the last two indices, the total current is conserved as a consequence of the field equation [25]:

$$
\partial_{\rho}\left[h\left(\dot{j}_{a}^{\rho}+\mathscr{T}_{a}^{\rho}\right)\right]=0
$$

In a purely spacetime form, the above field equation acquires the form

$$
\partial_{\sigma}\left(h \dot{S}_{\lambda} \rho \sigma\right)-k^{2}\left(\dot{h}_{\lambda}^{\rho}\right)=k^{2}\left(h \mathscr{T}_{\lambda}^{\rho}\right)
$$

where

$$
\dot{h}_{\lambda} \rho=\frac{h}{k^{2}}\left(\dot{\Gamma}^{\mu}{ }_{v \lambda} \dot{S}_{\mu}{ }^{\rho v}-\frac{1}{4} \delta_{\lambda}{ }^{\rho} \dot{T}^{\theta}{ }_{\mu \nu} \dot{S}_{\theta}^{\mu v}\right)
$$

is the energy-momentum pseudotensor of the gravitational field, and $\mathscr{T}_{\lambda} \rho=\mathscr{T}_{a}^{\rho} h^{a} \lambda$. It is important to notice that $\dot{t}_{\lambda} \rho$ is not simply the gauge current $\dot{j}_{a} \rho$ with the algebraic index " $a$ " changed to the spacetime index " $\lambda$ ". It incorporates also an extra term coming from the derivative term of the field equation:

$$
\dot{t}_{\lambda}^{\rho}=h_{\lambda}^{a} \dot{j}_{a}^{\rho}+k^{-2} \dot{\Gamma}_{\lambda v}^{\mu} \dot{S}_{\mu}^{\rho v}
$$

We see clearly from this equation the origin of the connection-term which transforms the gauge current $\dot{j}_{a} \rho$ into the energy-momentum pseudotensor $\dot{t}_{\lambda} \rho$.

Now, using the relation (15), it is possible to show that

$$
\dot{\mathscr{L}}=\stackrel{\circ}{\mathscr{L}}-\partial_{\mu}\left(2 h k^{-2} \dot{T}^{v \mu}{ }_{v}\right)
$$

where

$$
\stackrel{\circ}{\mathscr{L}}=-\frac{\sqrt{-g}}{2 k^{2}} \stackrel{\circ}{R}
$$

represents the Einstein-Hilbert Lagrangian of general relativity, with $\stackrel{\circ}{R}$ the scalar curvature of the Levi-Civita connection $\stackrel{\circ}{\Gamma}^{\rho} \mu v$. Up to a divergence, therefore, the teleparallel Lagrangian is equivalent to the Einstein-Hilbert Lagrangian of general relativity. It is important to observe also that, by using Eq. (15), the left-hand side of the field equation (25) can be shown to satisfy the relation

$$
\partial_{\sigma}\left(h \dot{S}_{\lambda} \rho \sigma\right)-k^{2}\left(\dot{h}_{\lambda} \rho\right)=h\left(\stackrel{\circ}{R}_{\lambda}^{\rho}-\frac{1}{2} \delta_{\lambda} \rho \stackrel{\circ}{R}\right) .
$$

This means that, as expected due to the equivalence between the corresponding Lagrangians, the teleparallel field equation (21) is equivalent to Einstein's field equation

$$
\stackrel{\circ}{R}_{\lambda}^{\rho}-\frac{1}{2} \delta_{\lambda} \rho \stackrel{\circ}{R}=k^{2} \mathscr{T}_{\lambda}^{\rho}
$$

We see in this way that, as already remarked, in spite of the conceptual differences between teleparallel gravity and general relativity, these theories are found to yield equivalent descriptions of gravitation. Although equivalent, however, they describe the gravitational interaction through a completely different mechanism. In the next section we are going to explore these differences.

\section{FORCE EQUATION VERSUS GEODESICS}

Let us consider, in the context of teleparallel gravity, the motion of a spinless particle of mass $m$ in a gravitational field $B^{a}{ }_{\mu}$. Analogously to the electromagnetic case [26], the action integral is written in the form

$$
\mathscr{S}=\int_{a}^{b}\left[-m c d \sigma-m c B^{a}{ }_{\mu} u_{a} d x^{\mu}\right],
$$


where $d \sigma=\left(\eta_{a b} d x^{a} d x^{b}\right)^{1 / 2}$ is the Minkowski tangent-space invariant interval,

$$
u^{a}=h_{\mu}^{a} u^{\mu}
$$

is the anholonomic particle four-velocity, with

$$
u^{\mu}=\frac{d x^{\mu}}{d s}
$$

the holonomic four-velocity, which is written in terms of the spacetime invariant interval $d s=\left(g_{\mu \nu} d x^{\mu} d x^{v}\right)^{1 / 2}$.

The first term of the action (32) represents the action of a free particle, and the second the coupling of the particle's mass with the gravitational field. Notice that the separation of the action in these two terms is possible only in a gauge theory, like teleparallel gravity, being not possible in general relativity. It is, however, equivalent with the usual action of general relativity. In fact, if we introduce the identities [19]

$$
h^{a}{ }_{\mu} u_{a} u^{\mu}=1
$$

and

$$
h^{a}{ }_{\mu} \frac{d \sigma}{d s}=\frac{\partial x^{a}}{\partial x^{\mu}},
$$

the action (32) can easily be seen to reduce to its general relativity version

$$
\mathscr{S}=-\int_{a}^{b} m c d s .
$$

In this case, the interaction of the particle with the gravitational field is described by the metric tensor $g \mu v$, which is present in $d s$.

Variation of the action (32) yields the equation of motion

$$
h_{\mu}^{a} \frac{d u_{a}}{d s}=\dot{T}^{a} \mu \rho u_{a} u^{\rho} .
$$

This is the force equation governing the motion of the particle, in which the teleparallel field strength $\dot{T}^{a} \mu \rho-$ that is, torsion - plays the role of gravitational force. To write it in a purely spacetime form, we use the relation

$$
h^{a}{ }_{\mu} \frac{d u_{a}}{d s}=\omega_{\mu} \equiv \frac{d u_{\mu}}{d s}-\dot{\Gamma}^{\theta}{ }_{\mu \nu} u_{\theta} u^{v}
$$

where $\omega_{\mu}$ is the spacetime particle four-acceleration. We then get

$$
u^{v} \dot{\nabla}_{v} u_{\mu} \equiv \frac{d u_{\mu}}{d s}-\dot{\Gamma}_{\mu \nu}^{\theta} u_{\theta} u^{v}=\dot{T}_{\mu \nu}^{\theta} u_{\theta} u^{v}
$$

The left-hand side of this equation is the Weitzenböck covariant derivative of $u_{\mu}$ along the world-line of the particle. The presence of the torsion tensor on its right-hand side, as already stressed, shows that in teleparallel gravity torsion plays the role of gravitational force. By using the identity

$$
\stackrel{\bullet}{T}_{\mu v}^{\theta} u_{\theta} u^{v}=-\stackrel{\bullet}{K}^{\theta}{ }_{\mu v} u_{\theta} u^{v}
$$

this equation can be rewritten in the form

$$
u^{v} \dot{D}_{\nu} u_{\mu} \equiv \frac{d u_{\mu}}{d s}-\left(\dot{\Gamma}_{\mu v}^{\theta}-\dot{K}_{\mu v}^{\theta}\right) u_{\theta} u^{v}=0 .
$$

The left-hand side of this equation is the teleparallel covariant derivative of $u_{\mu}$ along the world-line of the particle. Using the relation (15), it is found to be

$$
u^{v} \stackrel{\circ}{\nabla}_{v} u_{\mu} \equiv \frac{d u_{\mu}}{d s}-\stackrel{\circ}{\Gamma}^{\theta} \mu v u_{\theta} u^{v}=0 .
$$


This is precisely the geodesic equation of general relativity, which means that the trajectories followed by spinless particles are geodesics of the underlying Riemann spacetime. In a locally inertial coordinate system, the first derivative of the metric tensor vanishes, the Levi-Civita connection vanishes as well, and the geodesic equation (43) becomes the equation of motion of a free particle. This is the usual version of the (strong) equivalence principle as formulated in general relativity [27].

It is important to notice that the same principle holds in teleparallel gravity, but it operates differently. To see that, we use the torsion definition (12) to rewrite the force equation (40) in the form

$$
\frac{d u_{\mu}}{d s}-\dot{\Gamma}^{\theta}{ }_{v \mu} u_{\theta} u^{v}=0
$$

Observe that, as $\dot{\Gamma}_{\theta v \mu}$ is not symmetric in the last two indices, the left-hand side is not the covariant derivative of the four-velocity along the trajectory, and consequently it is not a geodesic equation. In other words, the trajectories followed by spinless particles are not geodesics of the underlying Weitzenböck spacetime. In a locally inertial coordinate system, the first derivative of the metric tensor vanishes, and the Weitzenböck connection $\dot{\Gamma}_{\theta v \mu}$ becomes skew-symmetric in the first two indices. In this coordinate system, therefore, owing to the symmetry of $u^{\theta} u^{v}$, the force equation (44) becomes the equation of motion of a free particle. This is the teleparallel version of the (strong) equivalence principle [18].

\section{MANAGING WITHOUT THE WEAK EQUIVALENCE PRINCIPLE}

Let us consider again the problem of the motion of a spinless particle in a gravitational field represented by the translational gauge potential $B^{a}{ }_{\mu}$. However, in order to explicitly violate the weak equivalence principle, we are going to assume that the gravitational mass $m_{g}$ and the inertial mass $m_{i}$ do not coincide. In this case, the action integral is written in the form

$$
\mathscr{S}=\int_{a}^{b}\left(-m_{i} c d \sigma-m_{g} c B^{a}{ }_{\mu} u_{a} d x^{\mu}\right) .
$$

We notice in passing that, due to the violation of the weak equivalence principle, this action cannot be reduced to the general relativity form, given by Eq. (37). Variation of (45) yields [19]

$$
\left(\partial_{\mu} x^{a}+\frac{m_{g}}{m_{i}} B^{a}{ }_{\mu}\right) \frac{d u_{a}}{d s}=\frac{m_{g}}{m_{i}} \dot{T}^{a}{ }_{\mu \rho} u_{a} u^{\rho}
$$

This is the force equation governing the motion of the particle, in which the teleparallel field strength $\dot{T}^{a}{ }_{\mu \rho}$ plays the role of gravitational force. Similarly to the electromagnetic Lorentz force, which depends on the relation $q / m_{i}$, with $q$ the electric charge of the particle, the gravitational force depends explicitly on the relation $m_{g} / m_{i}$ of the particle. In the Newtonian limit, this force equation reduces to the original Newton's law

$$
m_{i} \frac{d^{2} \vec{x}}{d t^{2}}=-m_{g} \vec{\nabla} \phi
$$

with $\phi=c^{2} B_{00}$ the gravitational potential [9]. It is important to observe that this limit is possible only because teleparallel and Newtonian gravity are both able to manage with the absence of the weak equivalence principle. It would be impossible in the context of general relativity.

The crucial point is to observe that, although the equation of motion depends explicitly on the relation $m_{i} / m_{g}$ of the particle, neither $B^{a}{ }_{\mu}$ nor $\dot{T}^{a}{ }_{\rho \mu}$ depends on this relation. This means essentially that the teleparallel field equation (21) can be consistently solved for the gravitational potential $B^{a}{ }_{\mu}$, which can then be used to write down the equation of motion (46), independently of the validity or not of the weak equivalence principle. Even in the absence of the weak equivalence principle, therefore, teleparallel gravity is able to describe the gravitational interaction [19].

Let us now see what happens in the context of general relativity. By using the identity (41), the force equation (46) can be rewritten in the form

$$
\frac{d u_{\mu}}{d s}-\stackrel{\circ}{\Gamma}^{\lambda} \mu \rho u_{\lambda} u^{\rho}=\left(\frac{m_{g}-m_{i}}{m_{g}}\right) \partial_{\mu} x^{a} \frac{d u_{a}}{d s},
$$


where use has been made also of the relation (15). Notice that the violation of the weak equivalence principle produces a deviation from the geodesic motion, which is proportional to the difference between the gravitational and inertial masses. ${ }^{3}$ Of course, when $m_{g}=m_{i}$, the equation of motion (48) reduces to the geodesic equation of general relativity. However, in the absence of the weak equivalence principle, it is not a geodesic equation, which means that it does not comply with the geometric description of general relativity, according to which the trajectories of all (spinless) particles must be given by geodesics. In fact, in the context of general relativity, there is no an action from where it can be obtained through a variational principle.

In order to comply with the foundations of general relativity, it is necessary to incorporate the particle properties into the geometry. This can be achieved by assuming, instead of the tetrad (9) of teleparallel gravity, the new tetrad

$$
\bar{h}^{a}{ }_{\mu}=\partial_{\mu} x^{a}+\frac{m_{g}}{m_{i}} B^{a}{ }_{\mu}
$$

which takes into account the characteristic $m_{g} / m_{i}$ of the particle under consideration. This tetrad defines a new spacetime metric tensor

$$
\bar{g}_{\mu v}=\eta_{a b} \bar{h}_{\mu}^{a} \bar{h}_{v}^{b}
$$

in terms of which the corresponding spacetime invariant interval is

$$
d \bar{s}^{2}=\bar{g}_{\mu \nu} d x^{\mu} d x^{v}
$$

By noticing that in this case the relation between the gravitational field strength and torsion turns out to be

$$
\frac{m_{g}}{m_{i}} T_{\mu \rho}^{a}=\bar{h}_{\lambda}^{a} \bar{T}_{\mu \rho}^{\lambda}
$$

it is an easy task to verify that, for a fixed relation $m_{g} / m_{i}$, the equation of motion (38) is equivalent to the true geodesic equation

$$
\frac{d \bar{u}_{\mu}}{d \bar{s}}-\bar{\Gamma}_{\mu \rho}^{\lambda} \bar{u}_{\lambda} \bar{u}^{\rho}=0
$$

where $\bar{u}_{\mu} \equiv d x_{\mu} / d \bar{s}=\bar{h}^{a}{ }_{\mu} u_{a}$, and $\bar{\Gamma}^{\rho}{ }_{\mu \nu}$ is the Christoffel connection of the metric $\bar{g}_{\mu \nu}$. Notice that this equation can also be obtained from the action integral

$$
\bar{S}=-m_{i} c \int_{a}^{b} d \bar{s}
$$

which is the usual form of the action in the context of general relativity.

However, the price for imposing a geodesic equation of motion to describe a non-universal interaction is that the gravitational theory becomes inconsistent. In fact, the solution of the corresponding Einstein's field equation

$$
\bar{R}_{\mu \nu}-\frac{1}{2} \bar{g}_{\mu \nu} \bar{R}=\frac{8 \pi G}{c^{4}} \overline{\mathscr{T}}_{\mu \nu},
$$

with $\overline{\mathscr{T}}_{\mu v}=\delta \mathscr{L}_{m} / \delta \bar{g}^{\mu v}$, would in this case depend on the relation $m_{g} / m_{i}$ of the test particle, which renders the theory inconsistent in the sense that test particles with different relations $m_{g} / m_{i}$ would require connections with different curvatures to keep all equations of motion given by geodesics. Of course, the gravitational field cannot depend on any test particle properties. We can then conclude that, in the absence of the weak equivalence principle, the geometric description provided by general relativity breaks down. Since the gauge potential $B^{a}{ }_{\mu}$ can always be obtained independently of any property of the test particle, teleparallel gravity remains as a consistent theory in the lack of universality.

\footnotetext{
3 Notice that, due to the assumed non-universality of free fall, it is not possible to find a local coordinate system in which the equation (48) reduces to the equation of motion of a free particle. This is a consequence of the fact that a violation of the weak equivalence principle precludes the existence of a strong version of the principle.
} 


\section{CONCLUSIONS}

One of the fundamental problems of gravitation is the inconsistency of Einstein's general relativity with quantum mechanics. Technically, it usually shows up as the impossibility of obtaining a renormalizable quantum theory for gravitation. However, there are conceptual reasons behind such inconsistency, essentially related to the very principles on which these theories are based. General relativity, as is well known, is based on the equivalence principle, whose strong version establishes the local equivalence between gravitation and inertia. Quantum mechanics, on the other hand, is fundamentally based on the uncertainty principle, which is a nonlocal principle. On this fundamental difference lies one of the roots of the difficulty in reconciling these two theories [2].

Now, although equivalent to general relativity, the gauge approach of teleparallel gravity is able to describe gravitation in a consistent way without resorting to the equivalence principle. The crucial point is the different character of the fundamental field of each theory: whereas in general relativity it is a tetrad field $h^{a}{ }_{\mu}$ (or equivalently, a metric tensor $g_{\mu \nu}$ ), in teleparallel gravity it is a gauge potential $B^{a}{ }_{\mu}$, the nontrivial part of the tetrad field:

$$
h^{a}{ }_{\mu}=\partial_{\mu} x^{a}+B^{a}{ }_{\mu}
$$

This apparently small difference has deep consequences. In fact, any gravitational theory whose fundamental field is a tetrad (or a metric) is necessarily a geometrical theory. On the other hand, a theory whose fundamental field is a gauge potential has not the same geometrical character. As a gauge theory it can, similarly to Maxwell's theory, be formulated independently of any equivalence principle. To understand this point, let us consider a particle whose gravitational mass $m_{g}$ does not coincide with its inertial mass $m_{i}$. Of course, both the weak and the strong equivalence principles are no longer valid. In this case, as we have seen, a geometrical theory for gravitation would require the introduction of a new tetrad field, given by [19]

$$
\bar{h}^{a}{ }_{\mu}=\partial_{\mu} x^{a}+\frac{m_{g}}{m_{i}} B^{a}{ }_{\mu}
$$

Since the relation $m_{g} / m_{i}$ of the test particle appears "inside" the tetrad definition, any theory in which $\bar{h}^{a}{ }_{\mu}$ is the fundamental field will be inconsistent in the sense that particles with different relations $m_{g} / m_{i}$ would require different solutions of the gravitational field equations to keep a geometric description of gravitation, in which all trajectories are necessarily given by geodesics. On the other hand, we see from the tetrad (57) that the relation $m_{g} / m_{i}$ appears "outside" the gauge potential $B^{a}{ }_{\mu}$. This means essentially that, in this case, the gravitational field equations (21-22) can be consistently solved for $B^{a}{ }_{\mu}$ independently of any test-particle property. This is the fundamental reason for teleparallel gravity to remain as a viable theory for gravitation, even in the absence of universality. We can then conclude that, similarly to what happens in Maxwell's theory, which is also a gauge theory, teleparallel gravity does not require the existence of an equivalence principle to describe the gravitational interaction. ${ }^{4}$ The replacement of general relativity by teleparallel gravity, therefore, may lead to a conceptual reconciliation of gravitation with quantum mechanics. Accordingly, the quantization of the gravitational field may also appear much more consistent if considered in the teleparallel picture. This is, of course, an open question yet to be explored.

\section{ACKNOWLEDGMENTS}

The authors would like to thank FAPESP, CNPq and CAPES for financial support.

\section{REFERENCES}

1. C. W. Misner, K. S. Thorne and J. A. Wheeler, Gravitation, Freeman, New York, 1973.

2. R. Y. Chiao, "Conceptual tensions between quantum mechanics and general relativity: Are there experimental consequences?" in Science and Ultimate Reality, edited by J. D. Barrow et al, Cambridge University Press, Cambridge, 2003 [gr-qc/0303100].

3. R. Aldrovandi, P. B. Barros and J. G. Pereira, Found. Phys. 33, 545 (2003) [gr-qc/0212034].

\footnotetext{
${ }^{4}$ We observe that, from the point of view of teleparallel gravity, the question of the existence or not of a quantum version of the weak equivalence principle becomes irrelevant.
} 
4. For a critical discussion of the equivalence principle, see T. Damour, Questioning the Equivalence Principle, contribution to the workshop Missions spatiales en physique fondamentale (Chatillon, France, 18-19/1/2001), edited by C. Bordé and P. Touboul, published in Comptes Rendus de l'Academie des Sciences (Serie IV) 2, 1249 (2001) [gr-qc/0109063].

5. J. L. Synge, Relativity: The General Theory, North-Holland, Amsterdam, 1960.

6. The present experimental status of gravitation can be found in C. M. Will, Living Rev. Rel. 4, 4 (2001) [gr-qc/0103036].

7. L. Viola and R. Onofrio, Phys. Rev. D55, 455 (1977); G. Z. Adunas, E. Rodriguez-Milla and D. V. Ahluwalia, Gen. Rel. Grav. 33, 183 (2001) [gr-qc/0006022]; A. Herdegen and J. Wawrzycki, Phys. Rev. D66, 044007 (2002) [gr-qc/0110021].

8. R. Colella, A. W. Overhauser and S. A. Werner, Phys. Rev. Lett. 34, 1472 (1974).

9. R. Aldrovandi, J. G. Pereira and K. H. Vu, Class. Quantum Grav. 21, 51 (2004) [gr-qc/0310110].

10. R. Aldrovandi, J. G. Pereira and K. H. Vu, "Selected Topics in Teleparallel Gravity", Proceedings of the 24th National Meeting of the Brazilian Physical Society, Caxambu, Brazil, 2003, edited by L. A. Ferreira, published in Braz. J. Phys. 34, 1374 (2004) [gr-qc/0312008].

11. D. Greenberger, Ann. Phys. (NY) 47, 116 (1968); D. Greenberger and A. W. Overhauser, Rev. Mod. Phys. 51, 43 (1979).

12. C. Lämmerzahl, Gen. Rel. Grav. 28, 1043 (1996) [gr-qc/9605065]; see also C. Lämmerzahl, Class. Quantum Grav. 15, 13 (1998) [gr-qc/9805009]; M. P. Haugan and C. Lämmerzahl, Lect. Notes Phys. 562, 195 (2001) [gr-qc/0103067].

13. Different phenomena have been named "gravitational Aharonov-Bohm effect" in the literature. See, for example, P. O. Mazur, Phys. Rev. Lett. 57, 929 (1986); P. O. Mazur, Phys. Rev. Lett. 59, 2380 (1987); A. K. Lawrence, D. Leiter and G. Samozi, Nuovo Cimento B17, 113 (1973); L. H. Ford and A. Vilenkin, J. Phys. A14, 2353 (1981); V. B. Bezerra, Class. Quant. Grav. 8, 1939 (1991).

14. F. W. Hehl, J. D. McCrea, E. W. Mielke and Y. Ne'emann, Phys. Rep. 258, 1 (1995).

15. Yu. N. Obukhov and J. G. Pereira, Phys. Rev. D67, 044016 (2003) [gr-qc/0212080].

16. R. Weitzenböck, Invariantentheorie, Noordhoff, Gronningen, 1923.

17. V. C. de Andrade, L. C. T. Guillen and J. G. Pereira, Phys. Rev. D64, 027502 (2001).

18. V. C. de Andrade and J. G. Pereira, Phys. Rev. D56, 4689 (1997) [gr-qc/9703059].

19. R. Aldrovandi, J. G. Pereira and K. H. Vu, Gen. Rel. Grav. 36, 101 (2004) [gr-qc/0303106].

20. A description of the teleparallel-based Einstein's unification theory can be found in T. Sauer, Field equations in teleparallel spacetime: Einstein's 'Fernparallelismus' approach towards unified field theory [physics/0405142]. For a historical account, see L. O'Raifeartaigh and N. Straumann, Rev. Mod. Phys. 72, 1 (2000) [hep-ph/9810524]; see also L. O'Raifeartaigh, The Dawning of Gauge Theory, Princeton University Press, Princeton, 1998.

21. See, for example, R. Aldrovandi and J. G. Pereira, An Introduction to Geometrical Physics, World Scientific, Singapore, 1995.

22. C. Møller, The Theory of Relativity, 2nd edition, Clarendon Press, Oxford, 1966.

23. J. W. Maluf, J. Math. Phys. 35, 335 (1994).

24. V. C. de Andrade, L. C. T. Guillen, and J. G. Pereira, Phys. Rev. Lett. 84, 4533 (2000) [gr-qc/0003100].

25. See, for example, N. P. Konopleva and V. N. Popov, Gauge Fields, Harwood, Chur, 1981.

26. L. D. Landau and E. M. Lifshitz, The Classical Theory of Fields, 3rd edition, Pergamon Press, New York, 1971.

27. S. Weinberg, Gravitation and Cosmology, Wiley, New York, 1972. 
Copyright of AIP Conference Proceedings is the property of American Institute of Physics and its content may not be copied or emailed to multiple sites or posted to a listserv without the copyright holder's express written permission. However, users may print, download, or email articles for individual use. 